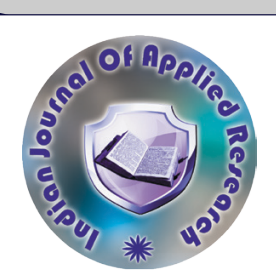

Periodontology

\title{
REGENERATIVE/RESECTIVE APPROACH FOR GRADE III FURCATION INVOLVEMENT- A CASE REPORT WITH QUESTIONABLE CLINICAL SCENARIO
}

\section{Valliammai \\ Rajendran}

\section{David}

Kadakampally*
Ex-Postgraduate student, M.D.S Periodontology., Department of Periodontology, Manipal College Of Dental Sciences, Mangalore, Manipal Academy of Higher Education (MAHE), Manipal-576104.

Associate Professor, M.D.S. Periodontology, Department of Periodontology, Manipal

College Of Dental Sciences, Mangalore, Manipal Academy of Higher Education (MAHE), Manipal-576104. *Corresponding Author Nagar, Madinaguda 500050.

Professor, M.D.S. Periodontology, Department of Periodontology, Manipal College Of Dental Sciences, Mangalore, Manipal Academy of Higher Education (MAHE), Manipal 576104.

\section{Deepa G Kamath}

\section{Ravalika N Kothuri}

Researcher, M.D.S. Public Health Dentistry, Dr. Sekhar's Dental Care and Implant Centre, Hyderabad, 500038.

\section{ABSTRACT}

Introduction: Teeth with furcation involvement can be maintained in a state of function for a longer period of time if appropriately treated and if the patient is motivated adequately. This report highlights the effect of regenerative and resective therapy in the management of grade III furcation involvement.

Observation: A 48 years old male patient was evaluated for furcation involvement with respect to the root canal treated lower right first molar tooth. Grade III furcation involvement was noted for which regenerative therapy was attempted with platelet rich fibrin (PRF) and bone grafts After 2 years of follow up, the furcation involvement still persisted and patient had a complaint of food lodgement. Hence, resective osseous surgery (tunnel preparation) was done to make the furcation ease for maintaining oral hygiene.

Commentary: In cases of periodontal loss, periodontal regenerative therapy must be the first treatment of choice. However, regeneration of grade III furcation involvement is more challenging and very few reports are available in the literature.

Conclusion: The selection of cases for regenerative or resective therapy depends on the clinicians' knowledge and practice. The ultimate goal of periodontal therapy is to provide a dentition that will function in health and comfort for life

\section{KEYWORDS : furcation involvement; regeneration; periodontal surgery}

\section{INTRODUCTION}

The ultimate goal of periodontal therapy is to provide a dentition that will function in health and comfort for life. Management of Furcation Involvement (FI) is a difficult task for the clinician. The success rate of regeneration is inversely proportional to the grade (Glickman's grading system) of FI. Grade III FI poses a great challenge. ' Only few cases with quite favorable success rates have been reported till date. Hence, it turns out to be a big question for the clinician whether to go for regenerative or resective approach. This article focuses on the management of grade III FI.

\section{Case Report}

\section{History}

A 48 years old male patient was referred to the outpatient Department of Periodontology for the evaluation of furcation defect with respect to the right lower $1^{\text {st }}$ molar. Patient had a history of pain in relation to the same tooth and visited the same institution, for which Root Canal Treatment (RCT) was done on lower right first molar. Patient had no medical/habit history.

\section{Periodontal Examination \& Procedure}

On periodontal examination, probing depth of $10 \mathrm{~mm}$ with grade III furcation involvement was noted (Table 1). Periapical radiograph was taken with respect to the concerned tooth as given in table 1. Consent was taken from the patient prior to any therapy. Non-surgical periodontal therapy was done comprising of scaling and root planing and oral hygiene instructions. The surgical procedure consisted of flap reflection both on buccal and lingual aspects to visualize $1-2 \mathrm{~mm}$ of healthy bone surrounding the defect and degranulation. PRF (Platelet Rich Fibrin) was obtained using the protocol given by Choukroun et $\mathrm{al}^{2}$ Intravenous blood from antecubital vein was collected in two $10 \mathrm{ml}$ sterile tubes and centrifuged at $3000 \mathrm{rpm}$ for 10 minutes. Later, PRF from first tube was made into a membrane by squeezing it with cotton gauze and PRF from second tube was mixed with decalcified freeze dried bone allograft (DFDBA) and packed into the defect. Flap was repositioned and sutured with 3-0 silk sutures (Figure 1). Periodontal

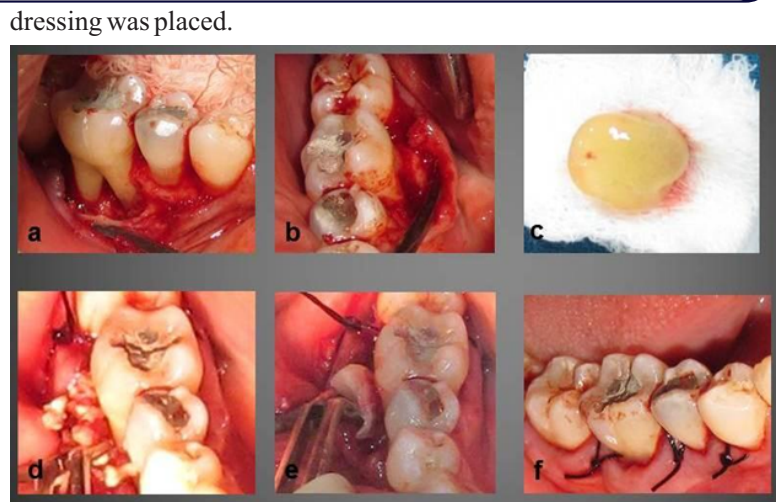

Figure 1: Regenerative Approach On Lower Right First Molar. a. Defect on buccal aspect; b. Defect on lingual aspect; c. PRF obtained; d. PRF and bone graft packed in the defect site; e. PRF membrane; f. Suturing

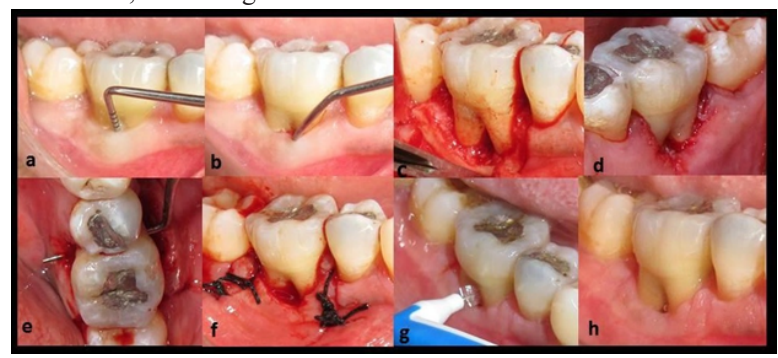

Figure 2: Tunneling Procedure On Lower Right First Molar.

a. Probing pocket depth; b. Furcation defect; c. Flap reflection on the buccal aspect;

d. Gingivectomy on the lingual aspect; e. Furcation tunnel; f. 
Suturing;

g. Application of interdental aid in the furcation tunnel; h. One year follow up

\section{Post-operative Care \& Follow Up}

Antibiotics and analgesics were prescribed. Oral hygiene in the surgical site was maintained with chlorhexidine mouthwash. Sutures and pack were removed 10 days postoperatively. Periodontal examination and maintenance was done at 1 and 3 months post operatively, every 6 months once thereafter.

At 2 years follow up, the periodontal examination showed recession in the furcation entrance, and persistent grade III furcation involvement. In addition, patient had a complaint of food lodgment in the furcation site. Radiographic examination revealed bone formation (radiopacity) at the mesial and distal aspects of lower right first molar, but at the furcation area there was still radiolucency depicting bone loss (Table 1).

Table 1: Comparison Of Clinical And Radiographic Parameters Between Baseline And 2 Years After Regenerative Therapy

\begin{tabular}{|l|l|l|}
\hline Parameters & At baseline & $\begin{array}{l}\text { 2 years after } \\
\text { regenerative } \\
\text { therapy }\end{array}$ \\
\hline Probing pocket depth & $10 \mathrm{~mm}$ & $6 \mathrm{~mm}$ \\
\hline Clinical attachment level & $12 \mathrm{~mm}$ & $10 \mathrm{~mm}$ \\
\hline Furcation involvement & Grade III & Grade III \\
\hline Mobility & Grade I & No mobility \\
\hline Clinical picture & & \\
& & \\
\hline Radiographic view & & \\
& & \\
& & \\
\hline
\end{tabular}

A tunnel preparation was planned to make the region accessible for oral hygiene maintenance. The procedure (Figure 2) consisted of apically positioned flap on the buccal aspect and gingivectomy on the lingual aspect. On the buccal aspect internal bevel gingivectomy of $1.5 \mathrm{~mm}$ was performed. Two vertical incisions on the mesial and distal line angles of the adjacent teeth were given and full thickness flap was reflected till the mucogingival junction. Degranulation was done. The flap was apically positioned and sutured with 3-0 silk sutures. Periodontal pack was placed. Sutures and pack removal were done at 1 week post operatively. Patient was advised to use an interdental brush in the furcation area and recalled every 2 weeks once for one month and once in every 6 months thereafter.

\section{DISCUSSION}

The clinician's decision in the management of a periodontal defect is significant, and it depends on various factors such as anatomic considerations, patient morbidity, etc. Goldman and Cohen stated that furcation defect is a no wall defect with poor prognosis due to lack of osteogenic cell proliferation, lack of sufficient bony walls providing the cells of the periodontal ligament which contribute most importantly to the regenerative process. ${ }^{3}$ In the present case report, regenerative approach in grade III FI did not result in satisfactory outcomes which had led to impaired oral hygiene maintenance care. The vertical bone loss of more than $3 \mathrm{~mm}$ in the furcation area limits the success rate of regenerative therapy ${ }^{4}$ and it was true in this case. A recent consensus report from AAP (American Academy of Periodontology) regeneration workshop on furcation defects stated that evidence of histologic periodontal regeneration in mandibular Class III defects is limited to one case report.

Apart from regenerative approach, various resective techniques are documented in the literature. This includes bicuspidization, hemisection and root resection. ${ }^{6-8}$ Bicuspidization is the separation of roots of mandibular molars along with its crown portion, after which both the segments are treated prosthetically and retained individually. This technique was not attempted in the present report as it results in deliberate removal of the tooth structure for the prosthetic management. In addition, it would further increase the expenditure for the patient. Hemisection/Root resection is indicated in cases of severe bone loss around one root of a tooth. Hence, it was not considered in the present treatment plan.

The tunneling procedure involves deliberate removal of bone to make it accessible for oral hygiene maintenance. However, in the present case, through and through FI (Grade III) was present and minimal bone was removed during the surgical procedure. The primary aim of the intervention was to make the furcation defect accessible to the patient for oral hygiene maintenance. Adequate oral hygiene care during the periodontal maintenance therapy might indicate that the tunneling procedure is a cost-effective alternative way for treating grade III FI. This procedure has few limitations such as root sensitivity and caries. However, its prevalence is limited to few cases. ${ }^{9,10}$ Long term follow up of a grade III furcation defect with a regenerative attempt followed by resective therapy makes this report unique in the literature.

\section{CONCLUSION}

The selection of cases for regenerative or resective therapy depends on the clinicians' knowledge and practice. Tunneling procedure showed a good periodontal maintenance in grade III FI cases. Only patients who are motivated for oral hygiene maintenance should be selected for tunneling procedure. However, further well conducted trials are needed to confirm the results obtained in the present report.

\section{Acknowledgement: None.}

\section{Financial support and sponsorship-None}

Conflicts of interest- There are no conflicts of interest.

\section{REFERENCES}

1. Glickman I. Clinical Periodontology. Philadelphia: WB Saunders Co., 1958, p. 694-6.

2. Choukroun J, Adda F, Schoeffl er C, Vervelle A. An opportunity in perio-implantology: The PRF. Implantodontie 2000;42:55-62.

3. Goldman HM, Cohen DW. The infrabony pocket. Classification and treatment. J Periodontol 1958;29:272-91

4. Pontoriero R, Lindhe J, Nyman S, Karring T, Rosenberg E, Sanavi F. Guided tissue regeneration in the treatment of furcation defects in mandibular molars: a clinical study of degree III involvements.J Clin Periodontol 1989;16:170-4.

5. Reddy MS, Aichelmann-reidy ME, Avila-ortiz G, Klokkevold PR, Murphy KG, Rosen PS et al. Periodontal Regeneration - Furcation Defects : A Consensus Report From the

6. Koduganti RR, Jandhyala S, Sandeep N, Reddy PVN. Effective management of mandibular Grade III furcation : A dicey issue. Joral Res Rev. 2014;6:21-5.

7. Niswade G, Bhutada G, Ansari S, Shende S. Management of furcation involved teeth- a case report! Int J Curr Adv Res. 2017;6:5018-21.

8. Nabhi K, Mehra P, Sharma B. Root Resection of Maxillary First Molar : A Case Repor . IOSR J Dent Med Sci. 2017;16:103-7.

9. Hamp SE, Nyman S, Lindhe J. Periodontal treatment of multirooted teeth: results after 5 years. J Clin Periodontol 1975;2:126-35.

10. Hellden LB, Elliot A, Steffensen B, Steffensen JE. The prognosis of tunnel preparations in treatment of Class III furcations: a follow-up study. J Periodontol 1989;60:182-7. 